\title{
Neurovascular unit on a chip: implications for translational applications
}

\author{
Donald J Alcendor, ${ }^{1,2}$, Frank E Block III2,3, David E Cliffel2,4, John Scott Daniels ${ }^{2,5}$, Kate LJ Ellacott ${ }^{2,6}$, Cody R Goodwin ${ }^{2,4}$,

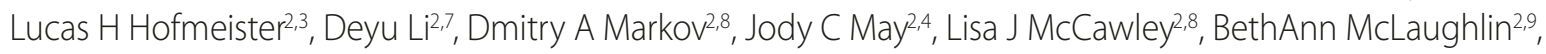 \\ John A McLean 2,4, Kevin D Niswender ${ }^{2,10}$, Virginia Pensabene2,3, Kevin T Seale2,3, Stacy D Sherrod ${ }^{2,11}$, Hak-Joon Sung ${ }^{2,3}$, \\ David L Tabb' 2,12 , Donna J Webb 2,13 and John P Wikswo*2,3,11
}

\begin{abstract}
The blood-brain barrier (BBB) dynamically controls exchange between the brain and the body, but this interaction cannot be studied directly in the intact human brain or sufficiently represented by animal models. Most existing in vitro BBB models do not include neurons and glia with other BBB elements and do not adequately predict drug efficacy and toxicity. Under the National Institutes of Health Microtissue Initiative, we are developing a threedimensional, multicompartment, organotypic microphysiological system representative of a neurovascular unit of the brain. The neurovascular unit system will serve as a model to study interactions between the central nervous system neurons and the cerebral spinal fluid (CSF) compartment, all coupled to a realistic blood-surrogate supply and venous return system that also incorporates circulating immune cells and the choroid plexus. Hence all three critical brain barriers will be recapitulated: blood-brain, brain-CSF, and blood-CSF. Primary and stem cell-derived human cells will interact with a variety of agents to produce critical chemical communications across the BBB and between brain regions. Cytomegalovirus, a common herpesvirus, will be used as an initial model of infections regulated by the BBB. This novel technological platform, which combines innovative microfluidics, cell culture, analytical instruments, bioinformatics, control theory, neuroscience, and drug discovery, will replicate chemical communication, molecular trafficking, and inflammation in the brain. The platform will enable targeted and clinically relevant nutritional and pharmacologic interventions for or prevention of such chronic diseases as obesity and acute injury such as stroke, and will uncover potential adverse effects of drugs. If successful, this project will produce clinically useful technologies and reveal new insights into how the brain receives, modifies, and is affected by drugs, other neurotropic agents, and diseases.
\end{abstract}

Keywords: brain-on-a-chip, microphysiological systems, blood-brain barrier, neurovascular unit, pericytes, microfluidic devices, cerebral spinal fluid, cytomegalovirus, neuropharmacology

\section{Introduction}

There is growing interest in the use of organs-on-chips or human vascular constructs [1-4] to mimic human physiology in a variety of clinical studies, including 'the assessment of drug or biologic candidate efficacy and toxicity' that has been cited by the National Institutes of Health (NIH) as a critical need for developing in vitro microphysiological systems [5]. In particular, the

*Corresponding author: john.wikswo@vanderbilt.edu

${ }^{2}$ Vanderbilt Institute for Integrative Biosystems Research and Education, Vanderbilt University, VU Station B 351807, Nashville, TN 37235-1807, USA Full list of author information is available at the end of the article development of drugs for treating disorders of the brain is severely limited by the lack of such systems to evaluate penetration of drugs into the brain [6]. Despite the physiological and pharmacological importance of the highly controlled chemical signaling between the systemic vascular system and the brain, there are only limited reports of the organ-on-a-chip approach being applied to the blood-brain barrier (BBB) [7-10].

Physical or pharmacological disruption of chemical signals between the systemic blood flow and the brain impairs normal functioning and responsiveness of the brain. Long-range chemical signaling through dysregulation of cytokines, nutrients, growth factors, hormones, lipids, neurotransmitters, drugs, and their metabolites is also important, but these chemical signals are difficult to 


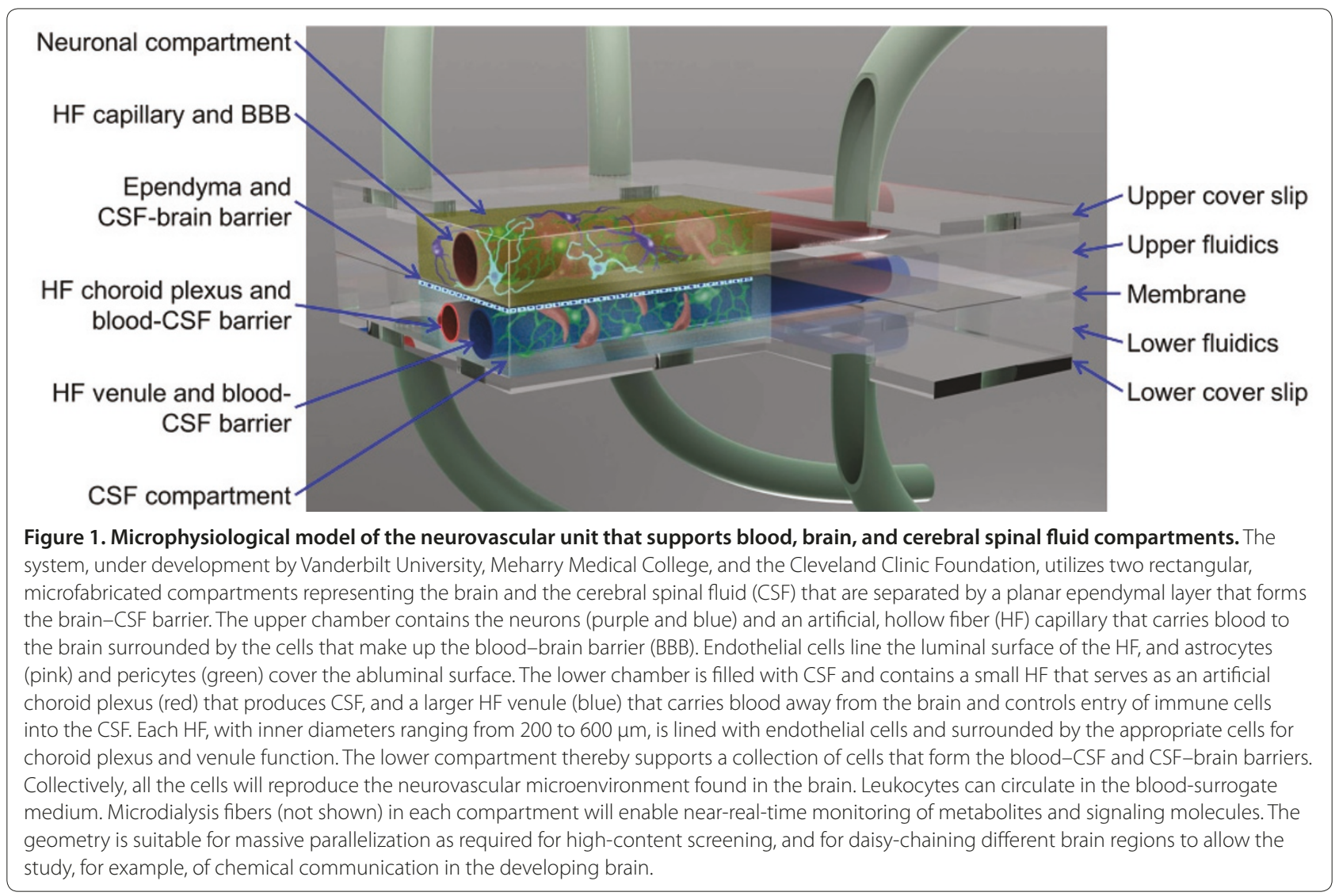

quantify and cells are typically studied in isolation. Hence there is a need for platforms that enable monitoring of complex intercellular communications. Recapitulating in vitro the physiological characteristics of brain vascular segments represents a critical issue. Adequate modeling of the cerebrovasculature could significantly help our understanding of the mechanisms and improve the pharmacology of diseases where the neurovascular interface is disrupted or pathologically altered. Moreover, the existing concern related to the use of animals (especially primates) has created additional pressure for a satisfactory in vitro BBB model.

As part of the NIH Microtissue Initiative, we are developing an in vitro, three-dimensional, multicompartment, organotypic neurovascular unit that includes a central nervous system (CNS) compartment coupled to a realistic BBB and blood-cerebral spinal fluid (CSF) barrier, circulating immune cells, and a CSF compartment (Figure 1). The neurovascular unit will ultimately be used with a recently developed multimodal analytical platform to examine the role of the BBB and the bloodCSF barrier in modulating chemical body-brain interactions and to characterize the interactions of astrocytes, pericytes, microglia, and neuronal and endothelial cells in the brain and its barriers. The unit will also assess the effect of a wide range of drugs, chemicals, and xenobiotics on the brain. The current and predicted clinical use of this model rests on its versatility to accommodate cells from patients with known pathologies who are (or are not) exposed to a drug treatment. The clinical viability of the BBB model upon which our approach is based has been demonstrated in rigorous comparison studies against human brain in situ [11]. Similarly, the microfabrication technology utilized in this device has proven robust for the study of neuron-neuron and neuron-glia interactions [12].

Our research should offer new and unbiased views of the correlations among the diverse chemical signals of the brain, enabled by a combination of state-of-the-art microfluidic devices, polymeric biomaterials, pump and valve systems, cell culture and organotypic human braincell preparations, analytical instruments such as on-chip miniature electrochemical sensors and off-chip ion mobility-mass spectrometers, computational bioinformatics techniques, and control theory that is needed to regulate the coupled organ systems $[4,13]$.

\section{Limitations of existing blood-brain barrier in vitro models}

The BBB comprises the interface between peripheral circulation and the CNS. As we have reviewed previously [14], the BBB not only supplies nutrients to the CNS and 
removes waste products, but also prevents blood-borne pathogens and toxic products from harming the brain. The molecular integrity of the $\mathrm{BBB}$ is the result of a network of tight junctions (TJs) between individual capillary endothelial cells that lack fenestration and have a reduced capacity for pinocytosis. The TJs of the capillary endothelium are supported by astrocyte end feet and pericytes. CNS pericytes are vital to BBB development [15] and function (they enhance TJ barrier function, stimulate expression of TJ proteins, and reduce permeability of the capillary endothelium to molecules passing between the cells $[16,17])$, and thus are a crucial element of the BBB model [18-20].

Most BBB in vitro culture models, however, include only brain microvascular endothelial cells (BMVECs) and astrocytes in a static Transwell culture. While the Transwell BBB system has been widely used owing to its simplicity, it does not provide the shear force critical for proper endothelial polarization and TJ formation [21], which result in both an endothelial permeability that is higher than physiological permeability and the absence of BBB-specific phenotypic characteristics [22]. Most important, current BMVEC/astrocyte models of the BBB do not take into account the close physical association of pericytes to the brain capillary endothelium [23]. The addition of intraluminal flow in a hollow fiber BBB model and the presence of astrocytes on the abluminal surface lead to physiologically realistic polarization of the endothelial cells and strengthen the TJs [11,24], which is a significant improvement over a static preparation. Flow-based microfluidic devices that co-culture endothelial and stromal cells produce an endothelium with physiologically appropriate polarization [11,25-27], but a well-studied and proven approach using hollow fibers does not allow visualization of the intraluminal cells $[11,21]$. Similarly, cell lines have been widely adapted to the Transwell model but, again, the results were far from being of translational relevance [22,28]. Many investigators have long recognized the limitations of immortalized cell lines or nonhuman cells and moved away from their use in favor of primary human cell cultures $[11,22,28,29]$. Our project under the NIH Microtissue Initiative seeks to address these shortcomings (Figure 2).

\section{Future needs, directions, and translational significance of an appropriate in vitro blood-brain barrier model}

The lack of an effective in vitro BBB model has a negative impact on pharmaceutical research and development, and subsequently affects the primary care of patients. At the cellular level, the BBB comprises BMVECs with TJs lining the brain microvessel, together with the closely associated astrocyte end-feet processes, where BMVECs

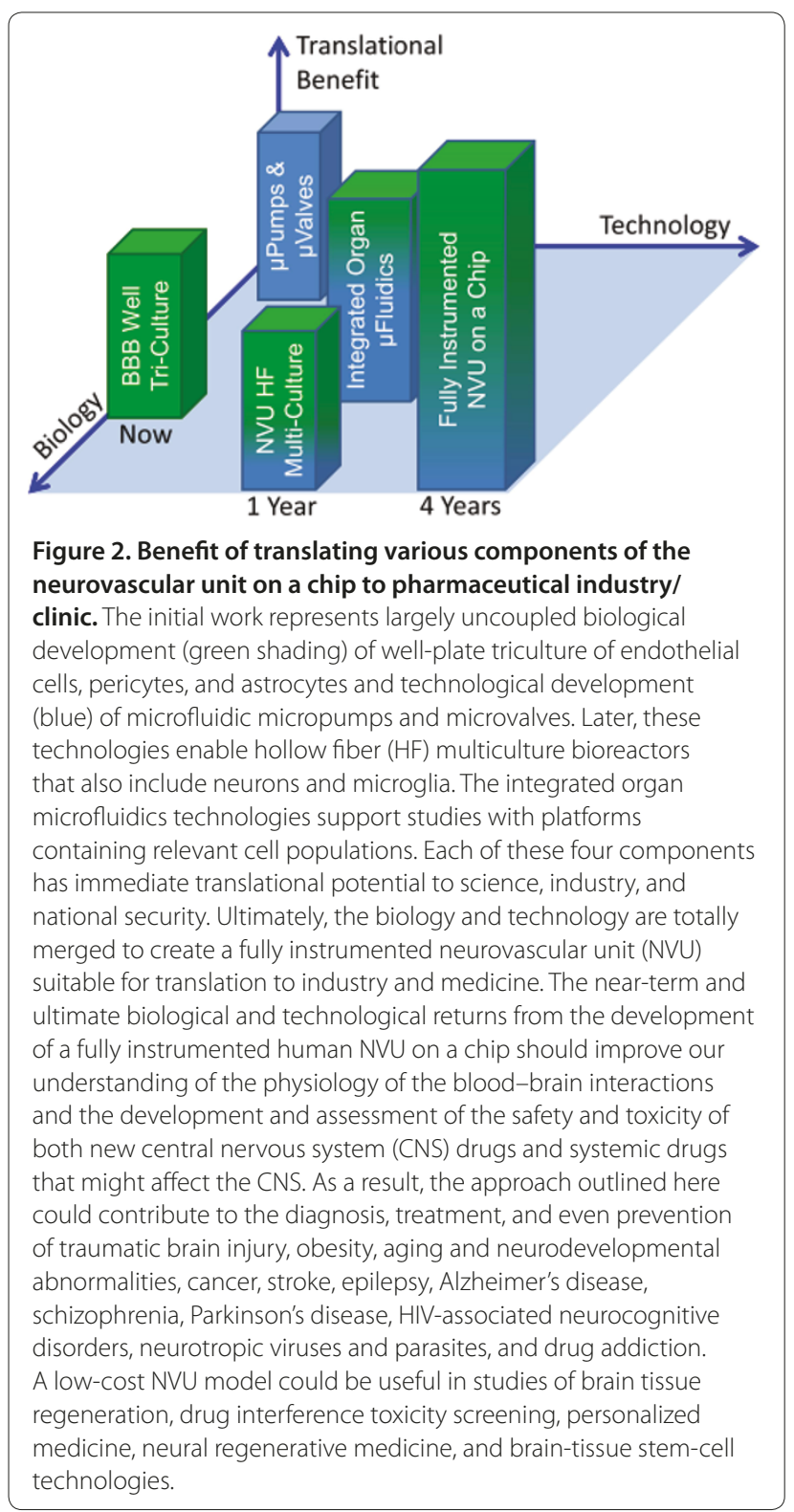

are responsible for the transport of xenobiotics, nutrients, hormones, and metabolites from the blood into the brain, and vice versa. The need for improved BBB models is even more apparent given the now-recognized role that pericytes play in the $\mathrm{BBB}$, particularly in infections such as human cytomegalovirus [14]. In addition, poor BBB penetration is responsible for the failure of several potentially useful CNS drugs to reach the market. Another important but often neglected issue in neuropharmacology is that proper function of the $\mathrm{BBB}$ enables the use of a broad spectrum of systemically active drugs that would be otherwise neurotoxic. This becomes clinically relevant since many diseases of the brain are associated with a leaky $\mathrm{BBB}$, and patients may be 
administered therapeutics that exert unanticipated harmful CNS effects (for example, use of antibiotics in advanced meningitis). Finally, the BBB provides a shield for exogenous neurotoxins spanning from nerve agents to psychotropic drugs, and hence a physiologically realistic BBB can be useful. Disturbances to the BBB have profound implications in traumatic brain injury, a topic of urgent interest to athletic organizations [30] and the military, yet are hard to study in vivo.

An effective in vitro $\mathrm{BBB} / \mathrm{CNS} / \mathrm{CSF}$ model such as we are working to develop must have a small fluid volume (lest the paracrine and metabolomic signals be diluted below physiological effect), require a limited number of human cells because of their cost and rarity, recreate interactions between different brain regions, allow visualization of cells on both sides of the $\mathrm{BBB}$, and be coupled in real time to advanced electrochemical and mass spectrometry instrumentation that can detect the important chemical signals [4]. The model's clinical utility would rest on its ability to recreate unique regions by selecting specific combinations of neurons, endothelial cells, astrocytes, other neuroglia, pericytes, and leukocytes. This model would use cells and fluids derived from patients with known pathologies to assess drug treatments and physiological stress from chronic diseases such as obesity, and acute injury such as stroke; would uncover potential adverse effects during drug discovery and phase 1 clinical trials, such as toxic transformation of approved drugs by brain endothelial cells; would detect novel and unbiased correlations between large numbers of chemical signals that converge at the BBB; and, finally, would combine microfluidic devices, pumps and valves, state-of-the-art cell culture, and organotypic human brain-cell preparations, analytical instruments, bioinformatics, control theory, and neuroscience drug discovery. The utilization of cells from individuals of different ethnic backgrounds would allow a mechanistic approach to understanding health disparities associated with drug metabolism that can often greatly influence clinical outcomes.

\section{Conclusion}

We are taking an integrated approach to the development of a detailed, in vitro model of the human neurovascular unit that should provide technologies applicable to clinical research that could reveal new mechanistic and region-specific insights into how the brain receives, metabolizes, and is otherwise affected by drugs, neurotropic agents, disease, and pathogens that traffic the BBB. We intend our platform to be implemented using primary human cells from normal or disease states obtained either commercially or from surgical resections; however, the ultimate contribution of in vitro microphysiological systems depends on the ability of either embryonic or induced pluripotent stem cells to provide the diversity of cell types required to support the widespread adoption of such human-like physiological models. Finally, our system is being designed to be linked to other organ-onchip systems supported by the NIH Microtissue Initiative.

\begin{abstract}
Abbreviations
BBB, blood-brain barrier; BMVEC, brain microvascular endothelial cell; CNS, central nervous system; CSF, cerebral spinal fluid; $\mathrm{NIH}$, National Institutes of Health; TJ, tight junction.
\end{abstract}

Competing interests

The authors declare that they have no competing interests.

\section{Acknowledgements}

Research reported in this publication was supported in part by the National Center for Advancing Translational Sciences of the NIH under Award No. UH2TR000491, NH Awards No. MH093903 and No. R21CA126728, and pilot grants from the Meharry Clinical and Translational Research Center (NIH Award No. U54RR026140), the Defense Threat Reduction Agency under Grants HDTRA1-09-1-00-13 and DTRA100271 A-5196, the Defense Advanced Research Projects Agency under Grant W911NF-12-2-0036, and a Vanderbilt University Interdisciplinary Discovery Grant. The content is solely the responsibility of the authors and does not necessarily represent the official views of the funding agencies and organizations. The authors thank Damir Janigro, Nicola Marchi, and Chaitali Ghosh for numerous conversations about the BBB. They thank Dominic Doyle for his help in preparing Figure 1, and Allison Price for her editorial assistance. The cost of publication of this manuscript was funded by the National Center for Advancing Translational Sciences of the NIH under Award No. UH2TR000491.

\section{Declarations}

Publication of this supplement has not been supported by sponsorship. Articles have undergone the journal's standard review process. The Editors declare that they have no competing interests.

This article has been published as part of Stem Cell Research \& Therapy Volume 4 Supplement 1, 2013: Stem cells on bioengineered microphysiological platforms for disease modeling and drug testing. The full contents of the supplement are available online at http://www.stemcellres.com/supplements/4/S1.

\section{Author details}

'Meharry Medical College, 1005 Dr. D. B. Todd Jr. Blvd, Nashville, TN 372083501, USA. ${ }^{2}$ Vanderbilt Institute for Integrative Biosystems Research and Education, Vanderbilt University, VU Station B 351807, Nashville, TN 37235 1807, USA. ${ }^{3}$ Department of Biomedical Engineering, Vanderbilt University, VU Station B 351631, Nashville, TN 37235-1631, USA. ${ }^{4}$ Department of Chemistry, Vanderbilt University, VU Station B 351822, Nashville, TN 37235-1822, USA. ${ }^{5}$ Center for Neuroscience Drug Discovery, Vanderbilt University Medical Center, 1205 Light Hall, Nashville, TN 37232-0697, USA. ${ }^{6}$ Department of Molecular Physiology and Biophysics, Vanderbilt University Medical Center, 702 Light Hall, Nashville, TN 37232-0615, USA. ${ }^{7}$ Department of Mechanical Engineering, Vanderbilt University, VU Station B 351592, Nashville, TN 372351592, USA. ${ }^{8}$ Department of Cancer Biology, Vanderbilt University Medical Center, 732 Preston Building, Nashville, TN 37232-6850, USA. ${ }^{\text {DDepartment }}$ of Neurology, Vanderbilt University Medical Center, 8110 MRB III, Nashville, TN 37232-8548, USA. ${ }^{10}$ Division of Diabetes, Endocrinology, and Metabolism, Vanderbilt University Medical Center, 7465 MRB IV, Nashville, TN 37232-0475, USA. "'Department of Physics and Astronomy, Vanderbilt University, VU

Station B 351807, Nashville, TN 37235-1807, USA. ${ }^{2}$ Department of Biomedical Informatics, Vanderbilt University Medical Center, U9211 MRB III, Nashville, TN 37232-8575, USA. ${ }^{13}$ Department of Biological Sciences, Vanderbilt University, VU Station B 1634, Nashville, TN 37235-1634, USA.

Published: 20 December 2013 


\section{References}

1. Esch MB, King TL, Shuler ML: The role of body-on-a-chip devices in drug and toxicity studies. Annu Rev Biomed Engr 2011, 13:55-72.

2. van der Meer $A D$, van den Berg $A:$ Organs-on-chips: breaking the in vitro impasse. Integr Biol 2012, 4:461-470.

3. Huh D, Torisawa YS, Hamilton GA, Kim HJ, Ingber DE: Microengineered physiological biomimicry: organs-on-chips. Lab Chip 2012, 12:2156-2164.

4. Wikswo JP, Block III FE, Cliffel DE, Goodwin CR, Marasco CC, Markov DA, McLean DL, McLean JA, McKenzie JR, Reiserer RS, Samson PC, Schaffer DK, Seale KT, Sherrod SD: Engineering challenges for instrumenting and controlling integrated organ-on-a-chip systems. IEEE Trans Biomed Eng 2013, 60:682-690

5. Integrated Microphysiological Systems for Drug Efficacy and Toxicity Testing in Human Health and Disease (UH2/UH3) [http://grants.nih.gov/grants/guide/rfa-files/RFA-RM-11-022.html]

6. Liu XR, Chen CP, Smith BJ: Progress in brain penetration evaluation in drug discovery and development. J Pharmacol Exp Ther 2008, 325:349-356.

7. Griep LM, Wolbers F, de Wagenaar B, ter Braak PM, Weksler BB, Romero IA, Couraud PO, Vermes I, van der Meer AD, van den Berg A: BBB ON CHIP: microfluidic platform to mechanically and biochemically modulate blood-brain barrier function. Biomed Microdevices 2013, 15:145-150.

8. Prabhakarpandian B, Shen MC, Nichols JB, Mills IR, Sidoryk-Wegrzynowicz M, Aschner M, Pant K: SyM-BBB: a microfluidic blood brain barrier model. Lab Chip 2013, 13:1093-1101.

9. Booth R, Kim H: Characterization of a microfluidic in vitro model of the blood-brain barrier ( $\mu$ BBB). Lab Chip 2012, 12:1784-1792.

10. Achyuta AKH, Conway AJ, Crouse RB, Bannister EC, Lee RN, Katnik CP, Behensky AA, Cuevas J, Sundaram SS: A modular approach to create a neurovascular unit-on-a-chip. Lab Chip 2013, 13:542-553.

11. Cucullo L, Marchi N, Hossain M, Janigro D: A dynamic in vitro BBB model for the study of immune cell trafficking into the central nervous system. J Cereb Blood Flow Metab 2011, 31:767-777.

12. Gao Y, Majumdar D, Jovanovic B, Shaifer C, Lin P, Zijlstra A, Webb D, Li D: $A$ versatile valve-enabled microfluidic cell co-culture platform and demonstration of its applications to neurobiology and cancer biology. Biomed Microdevices 2011, 13:539-548.

13. LeDuc PR, Messner WC, Wikswo JP: How do control-based approaches enter into biology? Annu Rev Biomed Engr 2011, 13:369-396.

14. Alcendor DJ, Charest AM, Zhu WQ, Vigil HE, Knobel SM: Infection and upregulation of proinflammatory cytokines in human brain vascular pericytes by human cytomegalovirus. J Neuroinflamm 2012, 9:95.

15. Daneman R, Zhou L, Kebede AA, Barres BA: Pericytes are required for blood-brain barrier integrity during embryogenesis. Nature 2010, 468:562-566.

16. Tsukita S, Furuse M, Itoh M: Multifunctional strands in tight junctions. Nat Rev Mol Cell Biol 2001, 2:285-293.
17. Ramsauer M, Krause D, Dermietzel R: Angiogenesis of the blood-brain barrier in vitro and the function of cerebral pericytes. FASEB J 2002, 16:1274-1276

18. Lai CH, Kuo KH: The critical component to establish in vitro BBB model: pericyte. Brain Res Rev 2005, 50:258-265.

19. Engelhardt B: Development of the blood-brain barrier. Cell Tissue Res 2003, 314:119-129.

20. Garberg P, Ball M, Borg N, Cecchelli R, Fenart L, Hurst RD, Lindmark T, Mabondzo A, Nilsson JE, Raub TJ, Stanimirovic D, Terasaki T, Oberg JO, Osterberg T: In vitro models for the blood-brain barrier. Toxicol In Vitro 2005, 19:299-334.

21. Cucullo L, Hossain M, Puvenna V, Marchi N, Janigro D: The role of shear stress in blood-brain barrier endothelial physiology. BMC Neurosci 2011, 12:40.

22. Santaguida S, Janigro D, Hossain M, Oby E, Rapp E, Cucullo L: Side by side comparison between dynamic versus static models of blood-brain barrier in vitro: a permeability study. Brain Res 2006, 1109:1-13.

23. Shepro D, Morel NML: Pericyte physiology. FASEB J 1993, 7:1031-1038.

24. Cucullo L, McAllister MS, Kight K, Krizanac-Bengez L, Marroni M, Mayberg MR, Stanness KA, Janigro D: A new dynamic in vitro model for the multidimensional study of astrocyte-endothelial cell interactions at the blood-brain barrier. Brain Res 2002, 951:243-254.

25. Huh D, Leslie DC, Matthews BD, Fraser JP, Jurek S, Hamilton GA, Thorneloe KS, MCAlexander MA, Ingber DE: A human disease model of drug toxicityinduced pulmonary edema in a lung-on-a-chip microdevice. Sci Trans/Med 2012, 4:159ra147.

26. Kim HJ, Huh D, Hamilton G, Ingber DE: Human gut-on-a-chip inhabited by microbial flora that experiences intestinal peristalsis-like motions and flow. Lab Chip 2012, 12:2165-2174

27. Huh D, Matthews BD, Mammoto A, Montoya-Zavala M, Hsin HY, Ingber DE: Reconstituting organ-level lung functions on a chip. Science 2010, 328:1662-1668.

28. Cucullo L, Hossain M, Rapp E, Manders T, Marchi N, Janigro D: Development of a humanized in vitro blood-brain barrier model to screen for brain penetration of antiepileptic drugs. Epilepsia 2007, 48:505-516.

29. Ghosh C, Marchi N, Desai NK, Puvenna V, Hossain M, Gonzalez-Martinez J, Alexopoulos AV, Janigro D: Cellular localization and functional significance of CYP3A4 in the human epileptic brain. Epilepsia 2011, 52:562-571.

30. Marchi N, Bazarian JJ, Puvenna V, Janigro M, Ghosh C, Zhong J, Zhu T, Blackman E, Stewart D, Ellis J, Butler R, Janigro D: Consequences of repeated blood-brain barrier disruption in football players. PloS One 2013, 8:e56805.

\section{doi:10.1186/scrt379}

Cite this article as: Alcendor DJ, et al.: Neurovascular unit on a chip: implications for translational applications. Stem Cell Research \& Therapy 2013, 4(Suppl 1):S18 